\title{
The influence of the electronic specific heat on swift heavy ion irradiation simulations of silicon
}

\author{
Galvin S. Khara \\ Department of Physics and Astronomy and The London Centre for \\ Nanotechnology, University College London, Gower Street, London WC1E 6BT, \\ United Kingdom \\ E-mail: galvin.khara.12@ucl.ac.uk
}

\section{Samuel T. Murphy}

Department of Physics and Astronomy and The London Centre for Nanotechnology, University College London, Gower Street, London WC1E 6BT, United Kingdom

E-mail: samuel.murphy@ucl.ac.uk

\section{Szymon L. Daraszewicz}

Department of Physics and Astronomy and The London Centre for Nanotechnology, University College London, Gower Street, London WC1E 6BT, United Kingdom

E-mail: szymon.daraszewicz.09@ucl.ac.uk

\section{Dorothy M. Duffy}

Department of Physics and Astronomy and The London Centre for Nanotechnology, University College London, Gower Street, London WC1E 6BT, United Kingdom

E-mail: d.duffy@ucl.ac.uk

\begin{abstract}
Swift heavy ion (SHI) irradiation of materials is often modelled using the two-temperature model. While the model has been successful in describing SHI damage in metals, it fails to account for the presence of a bandgap in semiconductors and insulators. Here we explore the potential to overcome this limitation by explicitly incorporating the influence of the bandgap in the parameterisation of the electronic specific heat for $\mathrm{Si}$. The specific heat as a function of electronic temperature is calculated using finite temperature density functional theory with three different exchange correlation functionals, each with a characteristic bandgap. These electronic temperature dependent specific heats are employed with two temperature molecular dynamics to model ion track creation in Si. The results obtained using a specific heat derived from density functional theory showed dramatically reduced defect creation compared to models that used the free electron gas specific heat. As a consequence, the track radii are smaller and in much better agreement with experimental observations. We also observe a correlation between the width of the band gap and the track radius, arising due to the variation in the temperature dependence of the electronic specific heat.
\end{abstract}


The influence of the electronic specific heat on swift heavy ion irradiation simulations of silicon 2

\section{Introduction}

Swift heavy ions (SHI) are fast heavy projectiles $(\geq 1 \mathrm{MeV} / \mathrm{amu})$, capable of depositing a highly localised density of energy in a target. Due to their low interaction cross section with the target nuclei, SHIs lose energy primarily by inelastic collisions with the electrons of the target material. This leads to a cylindrical region of excited electrons perpendicular to the SHI path. The electrons thermalise and energy is transferred to the nuclei via electron-phonon coupling, leading to localised heating and melting. As the energy is dissipated, defect recombination and recrystallisation occur, however, in certain materials a highly disordered region, called an ion track, can remain along the path of the SHI. Ion tracks have been experimentally observed in insulators $[1,2,3,4]$, semiconductors [5, 6, 7], and metals [8, 9].

The ability to precisely generate nanometre sized cylindrical defect regions in a material has a broad range of applications. For example, track-etched membranes are precisely modified polymers which have been irradiated by SHIs and treated with chemicals $[10,11]$. The band gaps of quantum wells and quantum dots can also be precisely modified via irradiation with SHIs $[12,13]$. It has also been shown that the critical current density for superconducting in Bi-2212 can be increased by a factor of 150 when irradiated by SHIs and subjected to a magnetic field. [14].

There are a number of models that attempt to describe the complex nonequilibrium physics during ion track formation. These include the Coulomb explosion model [15], where the SHI ionises the target. An alternative approach based on structural relaxation was suggested by Bennemann [16]. Here the bonding characteristic of the lattice is determined by the potential energy surface, and in regions of high electronic excitation this potential energy surface is modified. The most widely used model (and most likely hypothesis) is the inelastic thermal spike (iTS) model [17], where the SHI deposits energy into the electrons of a material, then this energy is coupled to the lattice via electron-phonon coupling, which subsequently causes the track formation. The most common way that the iTS model is formulated is using the two temperature model (TTM) [18]. In the two temperature model the electrons and lattice are described by two separate, but interacting, subsystems. This model has been widely used to simulate SHI irradiation in metals [19], semiconductors [20, 7, 21], and insulators[3, 4, 22].

Here we employ a variation of the TTM where we couple the electronic continuum subsystem to an MD supercell to generate a two temperature molecular dynamics $(2 \mathrm{~T}$ MD) simulation [23], where energy transfer by the electrons is represented using a heat diffusion equation. The 2T-MD approach allows energy transfer between the electron and ionic subsystem to represent electronic drag and electron-phonon coupling. The model was first developed for metals, although it has also been used to study ion track formation in band gap materials $[24,25,26]$. The ability of the model to describe bandgap materials is disputed, as it does not explicitly track the motion of carriers (i.e. electrons and holes) [27, 28]. Furthermore, the traditional free electron gas (FEG) model does not account for the energy required to cross the bandgap.

In this work we propose that through an appropriate parameterisation of the electronic specific heat the 2T-MD model may be employed for the study of bandgap materials. By calculating the electronic temperature dependent electronic heat capacity, $C\left(T_{e}\right)$, using density functional theory (DFT), we more accurately account for the band gap. In order to investigate the sensitivity of damage to $C_{e}(T)$ we use three different exchange correlation functionals as the widely used semi-local 
exchange correlation functionals lead to an underestimation of the bandgap of $\approx 20$ - $30 \%$. We employ a hybrid DFT functional that incorporates exact exchange from Hartree-Fock, which leads to a more accurate density of states (DOS) and hence a more accurate band gap. Using these electronic specific heats we perform 2T-MD simulations of SHI irradiation in silicon and compare the resulting track radii with similar results calculated with a specific heat from the commonly used free electron gas model $[3,29,20]$. This is particularly important as the specific heat has been shown to be a key parameter in determining whether defects multiply or anneal in metals [30].

\section{Methodology}

\subsection{Calculating the electronic specific heat}

The electronic specific heat capacity for silicon was found via finite temperature DFT $[31,32]$ using the Vienna Ab-initio Simulation Package software package (VASP) [33]. Simulations employed the semilocal Generalised Gradient Approximation (GGA) functional of Perdew, Burke, Ernzerhof (PBE) [34, 35], and the hybrid Heyd, Scuseria, and Ernzerhof (HSE) [36] and Perdew, Burke, Ernzerhof (PBE0) functionals [37]. Projector Augmented Wave (PAW) pseudo potentials were employed with the plane wave expansion truncated at $400 \mathrm{eV}$.

In all cases a 2 atom Si cell was used, with a 16 x 16x 16 Monkhorst-Pack [38] $k$-point grid for the integration of the Brillouin Zone. A Fock exchange grid of $16 \times 16$ $\mathrm{x} 16$ was chosen for HSE and PBE0 functionals and the mixing fraction of the Fock exchange for HSE was set to 0.25 , with a screening parameter of $0.2 \AA^{-1}$. Results for the ground state properties of these functionals are summarised in Table 1. These values are in good agreement with other published results using these functionals [39].

\begin{tabular}{ccc}
\hline Functional & Lattice Parameter $(\AA)$ & Band Gap $(\mathrm{eV})$ \\
\hline PBE & 5.469 & 0.71 \\
\hline HSE & 5.435 & 1.14 \\
\hline PBE0 & 5.430 & 1.84 \\
\hline Experiment & 5.431 & 1.10 \\
\hline
\end{tabular}

Table 1: Ground state DFT results of silicon compared with experiment [40, 41].

The electronic specific heat, $C_{e}$, for each functional was calculated using,

$$
C_{e}\left(T_{e}\right)=\frac{\partial U}{\partial T_{e}},
$$

where $U$ is the internal energy, and $T_{e}$ is the electronic temperature of the system. An electronic temperature was applied to the system via Fermi-Dirac smearing according to the formalism of Mermin [42] to an upper limit of 25,000 K. Self consistent field calculations were then carried out from $0 \mathrm{~K}$ to $25,000 \mathrm{~K}$ in increments of $250 \mathrm{~K}$, and the electronic specific heat was calculated using equation 1. 
The influence of the electronic specific heat on swift heavy ion irradiation simulations of silicon4

\subsection{Two Temperature Model}

As discussed in the Introduction SHI irradiation of Si is modelled using 2T-MD. Within the 2T-MD formalism energy transfer by the electrons is represented via heat diffusion equation as shown in equation 2 ,

$$
\frac{\partial T_{e}}{\partial t}=D_{e}\left(T_{e}\right) \nabla^{2} T_{e}-\frac{G}{C_{e}\left(T_{e}\right)}\left(T_{e}-T_{i}\right)+\frac{1}{C_{e}\left(T_{e}\right)} A\left(r\left[v_{i o n}\right], t\right),
$$

where $T_{e}$ and $T_{i}$ are the electronic and ionic temperatures respectively, $C_{e}\left(T_{e}\right)$ is the electronic specific heat, and $D_{e}\left(T_{e}\right)$ is the electronic diffusivity, which in silicon is $33.6 \mathrm{~cm}^{2} / \mathrm{s}$ at room temperature [43]. The spatial variation of the electronic diffusivity and electronic specific heat were ignored. We employed the temperature dependent diffusivity of Dufour et al in our simulations [44]. $G$ is the electron-phonon coupling term,

$$
G=\frac{3 N k_{B}}{m V \tau_{p}},
$$

where $N$ is the number of atoms in the relevant ionic cell, $k_{B}$ is the Boltzmann constant, $m$ is the mass of the atomic species, $V$ is the volume of the ionic cell, and $\tau_{p}$ is the electron-phonon relaxation time. $\tau_{p}$ was taken to be $0.26 \mathrm{ps}$, a value obtained from femtosecond optical pump probe reflectivity experiments [45]. $A\left(r\left[v_{i o n}\right], t\right)$ is a source term corresponding to the energy deposited by the SHI, and has a spatial dependence linked to the ion velocity, $v_{i o n}$, and temporal dependence $t$ described below.

In the 2T-MD model, the electronic subsystem is connected to the ions via a modified Langevin thermostat [23], and the ions evolve according to modified equations of motion,

$$
m_{i} \frac{\partial \mathbf{v}_{i}}{\partial t}=\mathbf{F}_{i}(t)-\gamma_{i} \mathbf{v}_{i}+\tilde{\mathbf{F}}_{i}(t),
$$

where $m$ and $\mathbf{v}_{i}$ are the mass and velocity of atom $i$ at time $t, \mathbf{F}_{i}(t)$ is the deterministic force on $i$ due to the interatomic potential. $\gamma_{i} \mathbf{v}_{i}$ is the frictional force that represents electronic drag, and $\gamma_{i}=\tau_{p}^{-1} . \tilde{\mathbf{F}}_{i}(t)$ is a stochastic force which is thermostatted at the electronic temperature. We employed the modified Tersoff potential for $\mathrm{Si}$ as derived by Kumagai et al [46] as it accurately reproduces the melting temperature, the latent heat of melting, the solid to liquid density change, and amorphous structure from quenched melting, which are all key parameters in the non equilibrium dynamics of SHI irradiation.

The simulations were run using a modified version of DL_POLY4 [47], on a 200,000 atom supercell measuring $271.99 \times 271.99 \times 54.4 \AA^{3}$ dimension (corresponding to an 8 atom cubic unit cell multiplied by $50 \times 50 \times 10$ ). This simulation volume was chosen as it ensures all the energy deposited into the electronic subsystem has a corresponding ionic cell to interact with. This MD cell was periodic in all directions to simulate a bulk crystal, and was subdivided into a grid of $25 \times 25 \times 5$ coarse grained ionic temperature voxels $\left(\right.$ each $\approx 10.86 \AA^{3}$ ). A $75 \times 75 \times 5$ electronic temperature voxel grid extends over the MD cell in the xy direction, and this system was solved using a space centred, forward in time Euler method with a timestep of 1 as. The MD system was pre-equilibrated for $200 \mathrm{ps}$ (with a 1 fs timestep) using an NPT ensemble (300 K) Nosé-Hoover thermo- and barostats. After equilibration the MD cell was connected to the continuum electronic system which was also set to $300 \mathrm{~K}$, and the SHI irradiation was simulated for $20 \mathrm{ps}$ (with a 1 fs timestep). 
The mean absorption radius of $0.74 \mathrm{~nm}$ was found using Bohr's principle of adiabatic variance [48]. We assume the SHI deposits energy via a spatial Gaussian and temporal exponential distribution with a characteristic deposition time of $1 \mathrm{fs}[49,50]$ (corresponding to a $C_{60}$ carbon cluster of a specific ion energy $0.07 \mathrm{MeV} / \mathrm{u}$ ). The source term was normalised so its spatial and temporal integration equates to the energy deposited into the electronic system (the electronic stopping power, $S_{e}$ ) $[49,28]$.

\section{Results and discussion}

Figure 1 shows the electronic specific heat for DFT simulations employing the different exchange correlation functionals compared with the free electron gas approximation. If one assumes that hot electrons in a band gap material behave like hot electrons in a metal [51] then the free electron gas model can be applied. For band gap materials this leads to an electronic specific heat $C_{e}=\frac{3}{2} n_{e} k_{B}$, where $n_{e}$ is the electron number density (taken to be one electron per atom [29]), and $k_{B}$ is the Boltzmann constant.

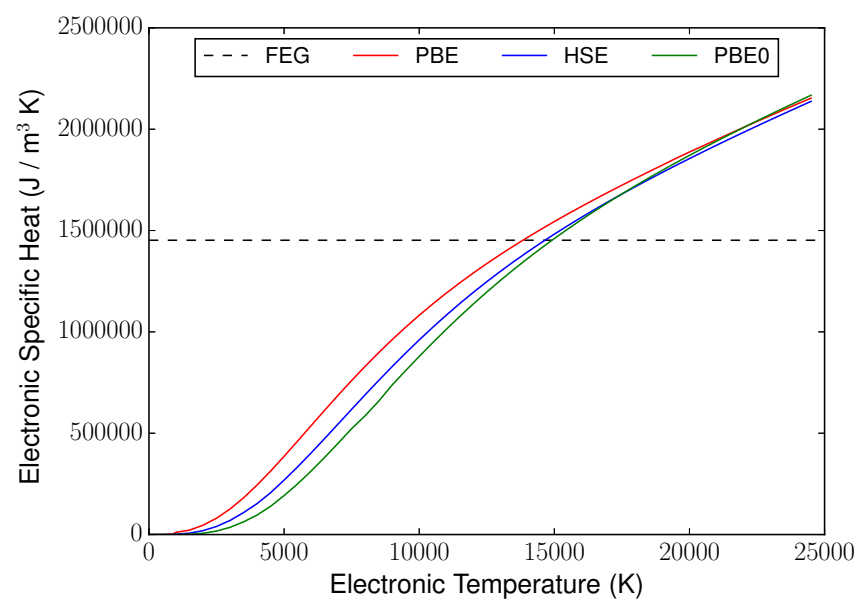

Figure 1: Electronic specific heat for Si, calculated using DFT with three different functionals compared to the free electron gas model.

The DFT results show a significant dependence of the specific heat on the choice of exchange correlation functional. As the curvatures of the DOS around the band edges are similar we conclude that the size of the bandgap is the dominant factor determining the specific heat. The larger the band gap, the higher the required electronic temperature for a non-zero internal energy, as the electrons need to first be excited across the band gap before they can subsequently contribute kinetically. Also, the larger the band gap, the lower the electronic specific heat. This can be explained by considering the DOS of our system, for a given amount of energy, a larger band gap results in occupancy of higher energy states, and thus a higher electronic temperature is achieved. Interestingly, at high electronic temperatures the electronic specific heats calculated using the three different functionals converge. These results show band gap contributions can indeed be accounted for within the 2T-MD model. 
Figure 2 shows the evolution of electronic and lattice temperatures at the centre of a typical SHI simulation for each $C_{e}\left(T_{e}\right)$. In general the SHI causes the electronic temperature to reach a maximum value in $10 \mathrm{fs}$, this energy is then transferred to the lattice, which reaches its maximum value within $0.1 \mathrm{ps}$, and both subsystems reach thermal equilibrium after a few ps. The difference between the temperatures predicted using the free electron gas and DFT specific heats are significant throughout. The FEG reaches a much higher maximum electronic temperature, cools at a slow constant rate, and reaches a significantly lower equilibrium temperature after a few ps. By contrast, the differences between functionals are less pronounced at the beginning and end of the simulation, but the regions where they diverge have a significant impact on the dynamics of the system.

The most striking differences in the temperature profiles for the different functionals occurs when the electronic temperature cools to between $20,000 \mathrm{~K}$ and $3,000 \mathrm{~K}$. As PBE has the highest $C_{e}\left(T_{e}\right)$ in this range, the rate of transfer to the lattice $\left(G / C_{e}\left(T_{e}\right)\right.$ in equation 2) is lower, thus electronic energy spreads further from the centre of the track before being transferred to the lattice. Conversely, a larger band gap leads to a lower $C_{e}\left(T_{e}\right)$, the rate of transfer to the lattice is higher, and thus the spatial region where the lattice temperature exceeds the melting threshold is more localised, leading to smaller track radii.

In a typical simulation a defect population is established within $0.1 \mathrm{ps}$. The number of defects increases as energy diffuses through the cell, reaching a maximum value at $1 \mathrm{ps}$. The lattice then cools, recrystallises, and a final defect distribution is established within 5 ps. Figures 3a - 3d show the evolution of local structure following SHI irradiation. Initially all atoms exhibit a perfect diamond structure (light blue), however, 0.1 ps after initialisation of the SHI there is a localised amorphous region at the centre of the simulation cell. The darker blue represents a denser region of cubic diamond which is due to the rapid expansion of the cylinder of atoms at the core. The black region is a small halo where diamond structure only extends out to the 1st and 2nd nearest neighbours. After 1 ps these compressed regions relax, and by $5 \mathrm{ps}$ the final defect distribution is observed. The Wigner-Seitz defect evolution at the corresponding times are shown in figures $3 \mathrm{e}-3 \mathrm{~h}$. The Wigner-Seitz defects were calculated using Voronoi cell analysis [52], if an atom moved from its original Voronoi cell a vacancy was formed, and if an atom moved into an occupied Voronoi cell an interstitial defect was formed.

Figure 4 shows the typical evolution of Wigner-Seitz defects for a low, middle, and high value of electronic stopping power. Each case displays the same qualitative behaviour, the number of defects reaches a maximum within $1 \mathrm{ps}$, after which about $10 \%$ of the defects recombine, and the final defect distribution is formed within 5 ps.

We performed a number of simulations to determine the relationship between the size of the ion track and the energy deposited by the SHI (quantified by the electronic stopping power, $\left.S_{e}\right)$ for each $C_{e}\left(T_{e}\right)$. We analysed the track distribution in two different ways, via track radius, and number of Wigner-Seitz defects. The track radius was determined by calculating the atomic density of each particle as a function of distance from the centre of cell using Voronoi cell analysis. Our results suggest that there are two distinct track radii profiles, which depend on the amount of energy deposited. At relatively low stopping powers the track consisted of an overly dense amorphous region, in figure 5 this corresponds to the blue line, which is an ion track of $\approx 18 \AA$. This is consistent with a mechanism whereby an overdense liquid core is created and subsequently quenched to form overdense amorphous Si. By contrast, at 
The influence of the electronic specific heat on swift heavy ion irradiation simulations of silicon 7

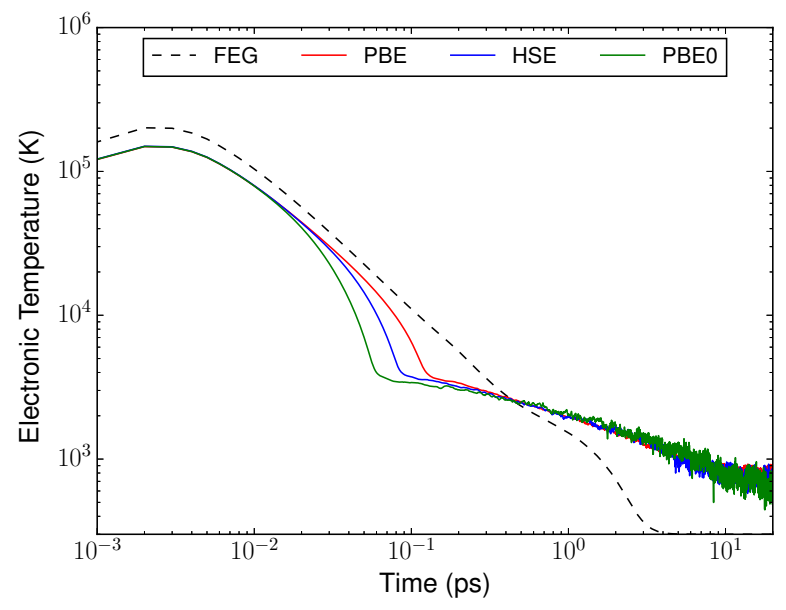

(a)

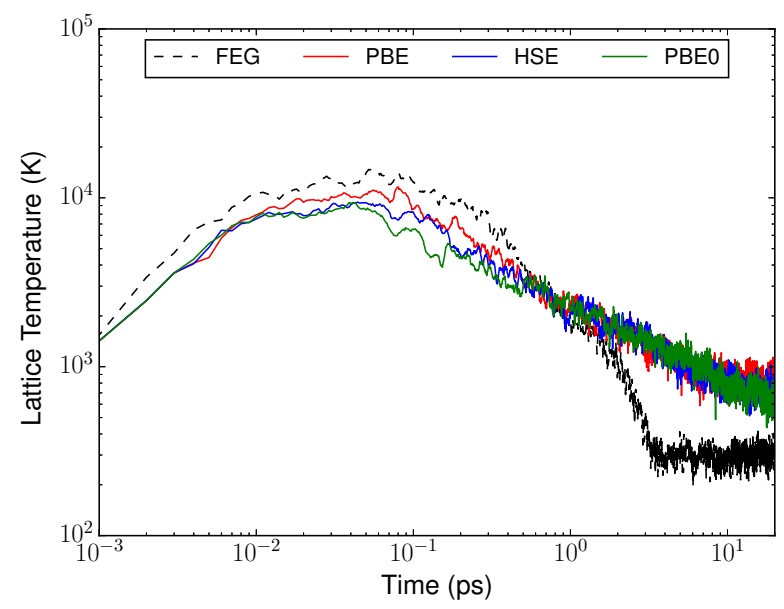

(b)

Figure 2: $25 \mathrm{keV} / \mathrm{nm}$ SHI irradiation temperature evolution of (a) the electronic system (b) the lattice, at the centre of the simulation cell using $C_{e}\left(T_{e}\right)$ calculated with different functionals.

higher stopping powers the energy deposition is great enough to eject atoms from the centre of the core. This results in an underdense core, surrounded by an overdense amorphous outer track region (shown by the red line in figure 5 , which is an ion track of $\approx 50 \AA$ ). These different track signatures may be observable in future small angle Xray scattering (SAXS) experiments on crystalline silicon. Previous SAXS experiments, and the corresponding MD simulations, that have characterised the damage resulting from SHI irradiation of amorphous Si [7] show an overdense core similar to our low 
The influence of the electronic specific heat on swift heavy ion irradiation simulations of silicon 8

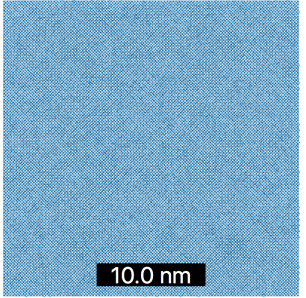

(a) $0.0 \mathrm{ps}$

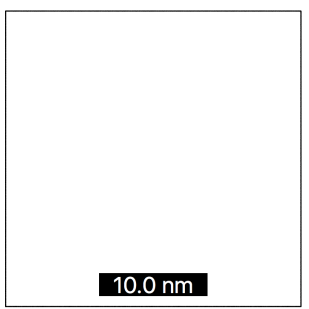

(e) $0.0 \mathrm{ps}$

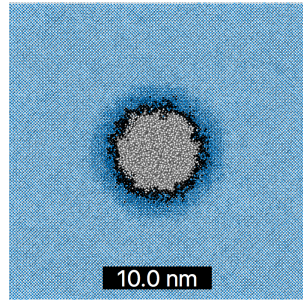

(b) $0.1 \mathrm{ps}$

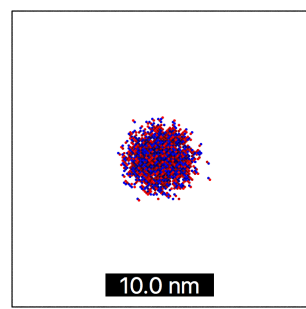

(f) $0.1 \mathrm{ps}$

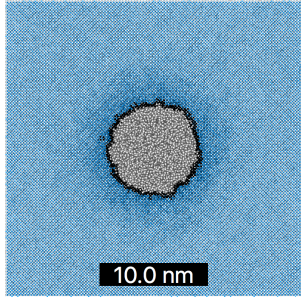

(c) $1.0 \mathrm{ps}$

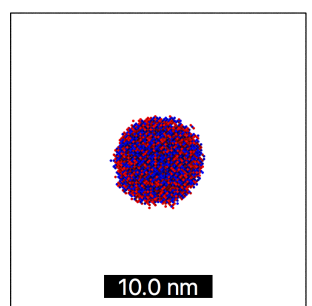

(g) $1.0 \mathrm{ps}$

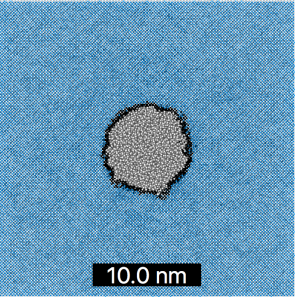

(d) $5.0 \mathrm{ps}$

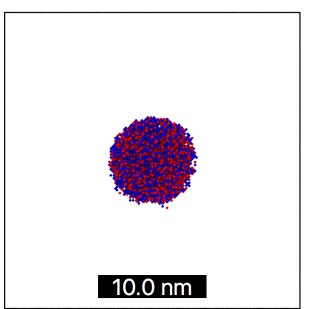

(h) $5.0 \mathrm{ps}$

Figure 3: [001] ion track morphology at various times during a $25 \mathrm{keV} / \mathrm{nm}$ SHI simulation. (a) - (d) are coloured according to local structure : light blue represents diamond structure, the darker blue is a region of compressed cubic diamond, black is 1st and 2nd neighbour diamond, and grey amorphous structure, determined via common neighbour analysis. (e) - (h) are Wigner-Seitz defects, blue representing vacancies and red representing interstitials. Both sets of images were created using OVITO [53].

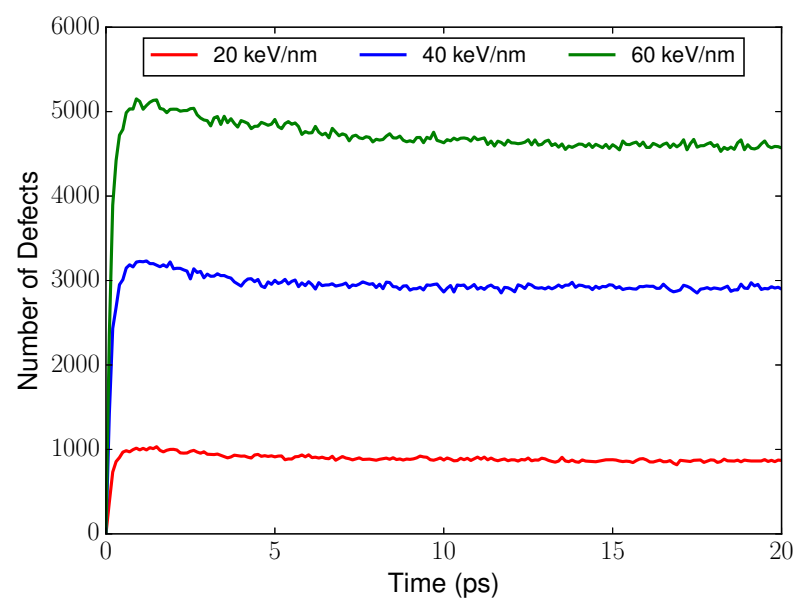

Figure 4: Evolution of Wigner-Seitz defects with time for various stopping powers using the electronic specific heat derived with the HSE functional 
The influence of the electronic specific heat on swift heavy ion irradiation simulations of silicon 9

stopping power result in figure 5.

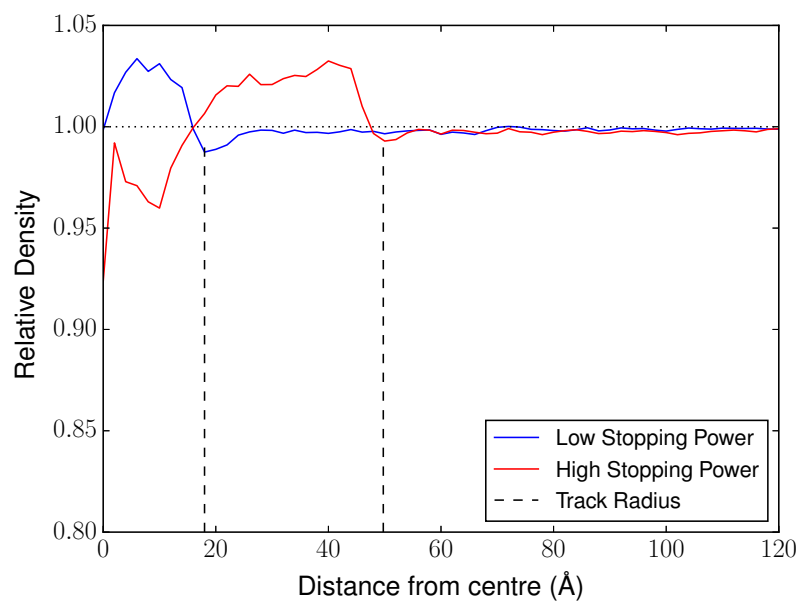

Figure 5: Relative atomic density as a function of distance from the centre of the simulation cell. The black dotted line corresponds to the ion track radius. Blue and red lines show the results from a $10 \mathrm{keV} / \mathrm{nm}$ and $25 \mathrm{keV} / \mathrm{nm}$ SHI simulations, using the PBE0 and PBE electronic specific heats respectively. The dotted vertical line marks the track radius of $18 \AA$ for the low stopping power and $50 \AA$ for the high stopping power.

Figure 6 shows how the electronic specific heat leads to significantly different final defect distributions and track radii. At $5 \mathrm{keV} / \mathrm{nm}$ we predict the formation of an ion track with a radius of $16.9 \AA$ using the specific heat determined by the free electron gas model. By contrast, no continuous ion tracks are formed when the specific heats from DFT are used, but there are some isolated vacancy and interstitial defects. Simulations using the free electron gas approximation were only carried out to an $S_{e}$ of $25 \mathrm{keV} / \mathrm{nm}$ as higher stopping powers resulted in defect distributions that approached the boundary of our MD cell. For all stopping powers tested the number of defects and the resulting track radii calculated using $C_{e}\left(T_{e}\right)$ from the free electron gas model are substantially greater than when using $C_{e}\left(T_{e}\right)$ determined via DFT.

Figure $6 \mathrm{~b}$ suggests that when DFT is used to parameterise the specific heat, the change in the track radius as a function of stopping power reproduces the relationship observed in experimental studies of other semiconductors $[5,6,7]$ and insulators $[1,2,3]$. The free electron model, on the other hand, shows a linear relationship between track radius and electronic stopping power. This is because the rate of energy transfer from the electrons to the lattice is constant regardless of electronic temperature, thus the spatial region of atoms which has a temperature higher than the melting threshold increases proportionally to the electronic stopping power.

A clear relationship can be observed between the sensitivity of ion track radii with different electronic specific heats, each with a characteristic density of states (and hence band gap). The larger the band gap, the more resistant the material is to damage (assuming all the other parameters in the model remain the same). An 


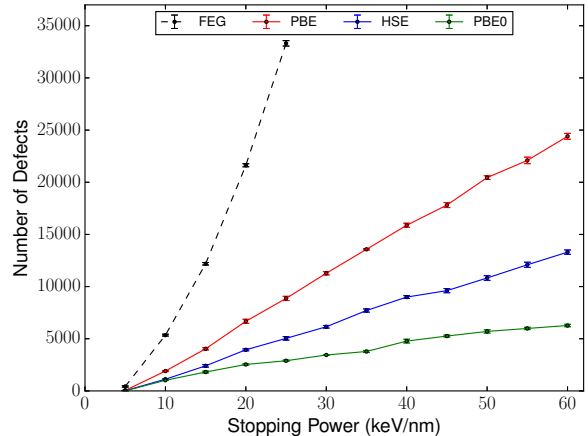

(a)

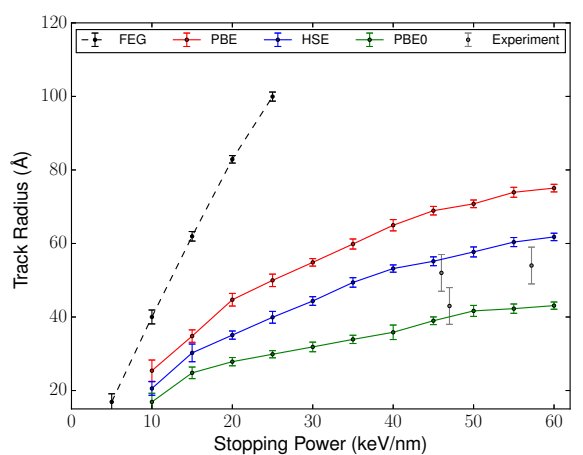

(b)

Figure 6: Defects vs $S_{e}$. (a) is the total number of Wigner-Seitz defects as a function of stopping power. (b) is the track radius as a function of stopping power. Each data point is the mean value of five repeated simulations, and the error bars correspond to the standard deviation.

apparent relationship between the threshold stopping powers and the band gap of a semiconductor has been noted previously from experimental observations [3]. This was attributed to an inverse relationship between the electron mean free path and the band gap, although no physical justification was offered. Here we claim that it is the electronic specific heat, as opposed to the mean free path, that is related to the band gap, and the modified relaxation time for the electron ion energy transfer affects the track radii.

The HSE functional predicts a bandgap in excellent agreement with the experimental value of $1.11 \mathrm{eV}$ [41]. Our track results with this functional also agree well with experimental tracks observed via the irradiation of silicon using $C_{60}$ fullerenes. Our threshold for track creation is lower than experimental observations [54, 55], but this may be explained by the fact that fast monatomic ions deposit energy more widely amongst the targets electrons, leading to less damage. We also note that we have not included the changes in the interatomic interactions due to electronic excitation [56, 57, 58], which may influence the results. The effects of these modified interactions will be investigated, using electronic temperature dependent potentials, in future work.

\section{Conclusion}

We have modelled the damage tracks resulting from swift heavy ion irradiation in silicon, using a 2T-MD model, for a range of stopping powers. We found a clear difference in track morphology for low stopping powers, where the track had an overdense core, and high stopping powers, where the tracks had a core shell structure with an underdense core and an overdense shell. We calculated the temperature dependence of the electronic specific heat, an important parameter of the 2T-MD model, using DFT with different functionals. These functionals have characteristic density of states (and thus different band gaps). This leads to distinct temperature dependent specific heats. The lower specific heat associated with larger band gaps 
The influence of the electronic specific heat on swift heavy ion irradiation simulations of silicon11

resulted in a shorter relaxation time for the electron lattice energy transfer and corresponding smaller track radii. This provides an explanation for the observed dependence of threshold stopping power on bandgap, which has previously been attributed to the mean free path. Simulations using the specific heat with the most accurate band gap give track radii close to the those observed in experiment.

In summary, we have demonstrated that bandgap effects can be introduced into the two temperature model by an accurate representation of the temperature dependence of the electronic specific heat.

\section{Acknowledgements}

GSK acknowledges funding from the European Office of Aerospace Research \& Development, STM acknowledges funding from the Leverhulme trust (grant number RPG2013-331). Via our membership of the UKs HPC Materials Chemistry Consortium, which is funded by EPSRC (EP/L000202), this work made use of the facilities of ARCHER, the UKs national high-performance computing service, which is funded by the Office of Science and Technology through EPSRCs High End Computing Programme. Additional computational resources were provided by the high performance computer cluster GRACE at UCL.

[1] Ishikawa N, Sonoda T, Sawabe T, Sugai H and Sataka M 2013 Nuclear Instruments and Methods in Physics Research, Section B: Beam Interactions with Materials and Atoms 314 180-184 ISSN 0168583X

[2] Afra B, Rodriguez M D, Trautmann C, Pakarinen O H, Djurabekova F, Nordlund K, Bierschenk T, Giulian R, Ridgway M C, Rizza G, Kirby N, Toulemonde M and Kluth P 2013 SAXS investigations of the morphology of swift heavy ion tracks in $\alpha$-quartz.

[3] Toulemonde M, Dufour C, Meftah A and Paumier E 2000 Nuclear Instruments and Methods in Physics Research, Section B: Beam Interactions with Materials and Atoms 166 903-912 ISSN 0168583X

[4] Lu F, Wang J, Lang M, Toulemonde M, Namavar F, Trautmann C, Zhang J, Ewing R C and Lian J 2012 Phys. Chem. Chem. Phys. 14 12295-12300

[5] Colder A, Canut B, Levalois M, Marie P, Portier X and Ramos S M M 2002 Journal of Applied Physics 91 5853-5857 ISSN 00218979

[6] Steinbach T, Bierschenk T, Milz S, Ridgway M C and Wesch W 2014 Journal of Physics D: Applied Physics 47065301 ISSN 0022-3727

[7] Bierschenk T, Giulian R, Afra B, Rodriguez M D, Schauries D, Mudie S, Pakarinen O H, Djurabekova F, Nordlund K, Osmani O, Medvedev N, Rethfeld B, Ridgway M C and Kluth P 2013 Physical Review B - Condensed Matter and Materials Physics 88174111 ISSN 10980121

[8] Dunlop A, Legrand P, Lesueur D, Lorenzelli N, Morillo J, Barbu A and Bouffard S 2007 Europhysics Letters (EPL) $\mathbf{1 5}$ 765-770 ISSN 0295-5075

[9] Barbu A, Dunlop A, Lesueur D and Averback R S 1991 Europhysics Letters 15 37-42 ISSN 0295-5075

[10] Apel P 2003 Nuclear Instruments and Methods in Physics Research, Section B: Beam Interactions with Materials and Atoms 208 11-20 ISSN 0168583X

[11] Vijay Y K 2009 Indian Journal of Physics 83 927-935 ISSN 0019-5480

[12] Choudhury, N and Singh, F and Sarma, B K N C 2013 Radiation effects and defects in solids 168 498-503 ISSN 1042-0150

[13] Devaraju G, Sathish N, Pathak A P, Turos A, Bazzan M, Trave E, Mazzoldi P and Arora B M 2010 Nuclear Instruments and Methods in Physics Research, Section B: Beam Interactions with Materials and Atoms 268 3001-3004 ISSN 0168583X

[14] Wiesner J, Fue $\beta$ H, Wirth G, Jäger E, Schimpf E, Wagner P, Hillmer F and Adrian H 1994 Physica C: Superconductivity 235-240 2971-2972 ISSN 09214534

[15] Fleischer R L, Price P B and Walker R M 1965 Journal of Applied Physics 36 3645-3652 ISSN 00218979

[16] Bennemann K H 2004 Journal of Physics: Condensed Matter 16 R995-R1056 ISSN 0953-8984

[17] Primak W 1955 Phys. Rev. 98 1854-1855 
The influence of the electronic specific heat on swift heavy ion irradiation simulations of silicon12

[18] Lifshits I, Kaganov M and Tanatarov L 1960 Journal of Nuclear Energy. Part A. Reactor Science 12 69-78 ISSN 03683265

[19] Wang Z G, Dufour C, Paumier E and Toulemonde M 1994 Journal of Physics: Condensed Matter 6 6733-6750 ISSN 0953-8984

[20] Chettah A, Kucal H, Wang Z, Kac M, Meftah A and Toulemonde M 2009 Nuclear Instruments and Methods in Physics Research Section B: Beam Interactions with Materials and Atoms 267 2719-2724 ISSN 0168583X

[21] Kamarou A, Wesch W, Wendler E, Undisz A and Rettenmayr M 2006 Physical Review B Condensed Matter and Materials Physics 73184107 ISSN 10980121

[22] Wang J, Lang M, Ewing R C and Becker U 2013 Journal of Physics: Condensed Matter 25 135001

[23] Duffy D M and Rutherford a M 2007 Journal of Physics: Condensed Matter 1916207 ISSN 0953-8984

[24] Leino A A, Daraszewicz S L, Pakarinen O H, Nordlund K and Djurabekova F 2015 EPL (Europhysics Letters) 11016004 ISSN 0295-5075

[25] Pisarev V V and Starikov S V 2014 Journal of physics. Condensed matter : an Institute of Physics journal 26475401 ISSN 1361-648X

[26] Phillips C L, Magyar R J and Crozier P S 2010 The Journal of chemical physics 133144711 ISSN 1089-7690

[27] Klaumunzer S 2006 Matematisk-fysiske Meddelelser 52306

[28] Daraszewicz S L and Duffy D M 2013 Nuclear Instruments and Methods in Physics Research, Section B: Beam Interactions with Materials and Atoms 303 112-115 ISSN 0168583X

[29] Toulemonde M, Assmann W, Dufour C, Meftah A, Studer F and Trautmann C 2006 Mat. Fys. Medd. Kong. Dan. Vid. Selsk. 52263

[30] Duffy D M, Itoh N, Rutherford a M and Stoneham a M 2008 Journal of Physics: Condensed Matter 20082201 ISSN 0953-8984

[31] Hohenberg P and Kohn W 1964 Phys. Rev. 136 B864 ISSN 01631829 (Preprint 1108.5632)

[32] Kohn W and Sham L J 1965 Physical Review 140 A1133-A1138 ISSN 0031899X

[33] Kresse G and Furthmüller J 1996 Physical Review B 54 11169-11186 ISSN 0163-1829

[34] Langreth D C and Mehl M J 1983 Physical Review B 28 1809-1834 ISSN 01631829 (Preprint 9909448)

[35] Perdew J P, Burke K and Ernzerhof M 1996 Phys. Rev. Lett. 77 3865-3868 ISSN 0031-9007 (Preprint 0927-0256(96)00008)

[36] Heyd J, Scuseria G E and Ernzerhof M 2003 Journal of Chemical Physics 118 8207-8215 ISSN 00219606

[37] Paier J, Hirschl R, Marsman M and Kresse G 2005 Journal of Chemical Physics 122234102 ISSN 00219606

[38] Monkhorst H J and Pack J D 1976 Physical Review B 13 5188-5192 ISSN 01631829 (Preprint arXiv: $1011.1669 \mathrm{v} 3)$

[39] Paier J, Marsman M, Hummer K, Kresse G, Gerber I C and Angyán J G 2006 Journal of Chemical Physics 124154709 ISSN 00219606

[40] Becker P, Scyfried P and Siegert H 1982 Zeitschrift für Physik B Condensed Matter 48 17-21 ISSN 14346036

[41] Precker J W and da Silva M a 2002 American Journal of Physics 701150 ISSN 00029505

[42] Mermin N D 1965 Physical Review 137 A1441-A1443 ISSN 0031899X (Preprint arXiv:1011.1669v3)

[43] Akkerman A and Murat M 2015 Nuclear Instruments and Methods in Physics Research, Section B: Beam Interactions with Materials and Atoms 350 49-54 ISSN 0168583X

[44] Dufour C, Khomenkov V, Rizza G and Toulemonde M 2012 Journal of Physics D: Applied Physics 45065302 ISSN 0022-3727 (Preprint arXiv:1111.0429v2)

[45] Sabbah A and Riffe D 2002 Physical Review B 66 1-11 ISSN 0163-1829

[46] Kumagai T, Izumi S, Hara S and Sakai S 2007 Computational Materials Science 39 457-464 ISSN 09270256

[47] Todorov I T, Smith W, Trachenko K and Dove M T Journal of Materials Chemistry 1911 ISSN 0959-9428

[48] Mozumder A 1974 The Journal of Chemical Physics 601145 ISSN 00219606

[49] Toulemonde M, Costantini J, Dufour C, Meftah a, Paumier E and Studer F 1996 Nuclear Instruments and Methods in Physics Research Section B: Beam Interactions with Materials and Atoms 116 37-42 ISSN 0168583X

[50] Gervais B and Bouffard S 1994 Nuclear Inst. and Methods in Physics Research, B 88 355-364 ISSN 0168583X 
The influence of the electronic specific heat on swift heavy ion irradiation simulations of silicon13

[51] Baranov I, Martynenko Y V, Tsepelevich S and Yavlinski Y N 1988 Soviet Physics Uspekhi 31 1015-1034 ISSN 0038-5670

[52] Rycroft C H, Grest G S, Landry J W and Bazant M Z 2006 Physical Review E - Statistical, Nonlinear, and Soft Matter Physics 74021306 ISSN 15393755 (Preprint 0602395)

[53] Stukowski A 2009 Modelling Simul. Mater. Sci. Eng. 18015012 ISSN 0965-0393

[54] Toulemonde M, Dural J, Nouet G, Mary P, Hamet J F, Beaufort M F, Desoyer J C, Blanchard C and Auleytner J 1989 Physica Status Solidi (a) 114 467-473 ISSN 00318965

[55] Mary P, Bogdanski P, Toulemonde M, Spohr R and Vetter J 1992 Nuclear Instruments and Methods in Physics Research Section B: Beam Interactions with Materials and Atoms 62 391-393 ISSN 0168583X

[56] Shokeen L and Schelling P K 2010 Applied Physics Letters 97151907 ISSN 00036951

[57] Shokeen L and Schelling P K 2011 Journal of Applied Physics 109073503 ISSN 00218979

[58] Murphy S T, Daraszewicz S L, Giret Y, Watkins M, Shluger A L, Tanimura K and Duffy D M 2015 Physical Review B - Condensed Matter and Materials Physics 92134110 ISSN $1550235 \mathrm{X}$ 